Cell Research (2003); 13(5):335-341

http://www.cell-research.com

\title{
Tissue engineering of blood vessels with endothelial cells differentiated from mouse embryonic stem cells
}

\author{
Gan Shen*, Hsiao Chien Tsung*, Chun Fang Wu, Xiao Yin Liu, Xiaoyun Wang, Wei Liu, Lei Cui, Yi Lin \\ $\mathrm{CAO} * *$
}

Department of Plastic and Reconstructive Surgery, Shanghai $9^{\text {th }}$ People's Hospital, Shanghai $2^{\text {nd }}$ Medical University, Shanghai Key Laboratory of Tissue Engineering, Shanghai 200011,China.

E-mail:YilinCao@sohu.com

\begin{abstract}
Endothelial cells $\left(\mathrm{TEC}_{3}\right.$ cells) derived from mouse embryonic stem (ES) cells were used as seed cells to construct blood vessels. Tissue engineered blood vessels were made by seeding $8 \times 10^{6}$ smooth muscle cells (SMCs) obtained from rabbit arteries onto a sheet of nonwoven polyglycolic acid (PGA) fibers, which was used as a biodegradable polymer scaffold. After being cultured in DMEM medium for 7 days in vitro, SMCs grew well on the PGA fibers, and the cell-PGA sheet was then wrapped around a silicon tube, and implanted subcutaneously into nude mice. After 6 8 weeks, the silicon tube was replaced with another silicon tube in smaller diameter, and then the $\mathrm{TEC}_{3}$ cells (endothelial cells differentiated from mouse ES cells) were injected inside the engineered vessel tube as the test group. In the control group only culture medium was injected. Five days later, the engineered vessels were harvested for gross observation, histological and immunohistochemical analysis. The preliminary results demonstrated that the SMC-PGA construct could form a tubular structure in 6 8 weeks and PGA fibers were completely degraded. Histological and immunohistochemical analysis of the newly formed tissue revealed a typical blood vessel structure, including a lining of endothelial cells (ECs) on the lumimal surface and the presence of SMC and collagen in the wall. No EC lining was found in the tubes of control group. Therefore, the ECs differentiated from mouse ES cells can serve as seed cells for endothelium lining in tissue engineered blood vessels.
\end{abstract}

Key words: tissue engineering, embryonic stem cell, blood vessel, differentiation, endothelial cell.

\section{INTRODUCTION}

In clinic, vascular pathological changes and damage are common diseases. These diseases are usually lifethreaten if they are not treated timely. The patients with vascular diseases often need transplantation of vascular grafts. Sometimes, autologous grafts may cause morbidity at the donor site. In addition, donated tissues are limited. Also, synthetic conduits made by high molecular compounds and allogeneic graft replacements have certain disadvantages that greatly limit their application to the long-term treatment. These conduits are foreign materials that lack the ability to grow, repair or remodel. More importantly, they are potentially thrombogenic. Therefore,

* Co-first authors. Dr. Gan SHEN now works in ZhongDa Hospital of Southeast University.

**Corresponding author. Fax:0086-21-53078128 E-mail: YilinCao@sohu com

Received Apr-4-2003 Revised Sep-15-2003 Accepted Sep-25-2003 tissue engineering might be a good approach to construct a blood vessel. However, tissue construction by tissue engineering approach needs a large amount of seed cells, such as endothelial cells. Thus, the finding of a source of large amount of human vascular endothelial cells is important to develop engineered vessels for the treatment of vascular diseases[1].

Weinberg and Bell first reported an approach for generating tissue engineered blood vessel in 1986[2].Since then many improved methods have been developed[35]. The effort to construct living blood vessels has recently been performed in our laboratory[6], using smooth muscle cells (SMCs) and endothelial cells (ECs) harvested from animal arteries and veins as seed cells. These cells were co-cultured with the PGA sheet in vitro for 1 week, then the PGA sheet were wrapped around a silicon tube to form a tubular structure and implanted sub- 
cutaneously in nude mice. After implantation for 6 weeks, the cell-PGA construct could form a tubular structure with an endothelial lining. But in this process, the autologous vessels must be harvested to isolate endothelial cells, but they were difficult to proliferate in culture. Besides, it also caused tissue damage for donors. Human embryonic stem (ES) cells have the potential to differentiate into various cell types[7] and thus, may provide a source of seed cells for tissue engineering. Differentiation of ES cells into endothelial cells and the formation of vessel structure have been studied extensively in murine ES cells including maturation steps, molecular events, and growth factor involvement[8-10]. Recently, Levenberg et al. described that human embryonic stem (hES) cells were able to differentiate into endothelial cells and form tubelike structures when cultured on matrigels. When transplanted into SCID mice, these cells appeared to form micro-vessels that contained mouse blood cells. Therefore, they concluded that these induced cells could provide a source of human endothelial cells for potential applications[11].

It is well known that endothelialization is important to maintain the long-term potency of small-caliber prosthetic blood vessels. Endothelial cells not only prevent thrombus formation but also secret some bio-factors into the blood[12-13], so endothelial cells play an important role in blood vessel. In previous studies, we have successfully induced mouse ES cells to differentiate into ECs, which were further immortalized by the transfection of human telomerase reverse transcriptase (hTERT) cDNA [14]. These immortalized differentiated cells were able to maintain the phenotype of ECs, including the expression of mRNA and proteins of fetal liver kinase-1 (flk1), von Willebrand factor (vWF) and CD34. These cells could form tubular structures in culture medium supplemented with vascular endothelial growth factor (VEGF), basic fibroblast growth factor (bFGF) and erythropoietin (EPO). In the present study, the induced ECs immortalized by hTERT cDNA were used as seed cells to engineer blood vessels. Using these induced ECs, we have successfully constructed several engineered blood vessels in the subcutaneous tissues in nude mice, and the engineered vessels contains an endothelial lining. Our preliminary results showed that it is possible to endothelialize engineered vessel with immortalized ECs differentiated from mouse ES cells.

\section{MATERIALS AND METHODS}

\section{$\mathrm{TEC}_{3}$ endothelial cells and the cell culture}

$\mathrm{TEC}_{3}$ cells were generated in our laboratory. Briefly, the embryonic bodies (EBs) formed in vitro from mouse ES7 (ES cell line also established in our laboratory used as parental cells ) cells were induced with retinoic acid (RA) at a concentration of 1 ? $0^{-9} \mathrm{~mol} / \mathrm{L}$ in Dulbecco抯 Modified Eagle Medium (DMEM, Gibco) containing 450 $\mathrm{mg} / \mathrm{L}$ glucose, 20\% FCS, $0.1 \mathrm{~mol} / \mathrm{L}$ b-mercaptoethanol, 1000 units $/ \mathrm{ml}$ leukemia inhibition factor (LIF) and 1\% nonessential amino acid (designated as Embryonic stem cells medium, ESM) for 5 days, then these EBs were attached to $6-\mathrm{cm}$ culture dishes coated with $0.1 \%$ gelatin, and cultured in DMEM medium containing 15\% FCS and 3 $\mathrm{ng} / \mathrm{ml}$ transforming growth factor- $\mathrm{b}_{1}\left(\mathrm{TGF}-\mathrm{b}_{1}\right)$. Following 2-5 days culture, some epithelial cells and a lot of round cells appeared around each of EBs. After 5-10 days culture, these 憆ound? cells were able to arrange into vascular tube-like structures. The results obtained by scanning electron microscopy and immunocytochemistry staining demonstrated that these tube-like structures were constituted by a large number of round cells, which were positive to vWF and CD34 antibodies (see also[]). Then these round cells were collected and transfected with the cDNA of human telomerase reverse transcriptase (hTERT) by Lipofectamine 2000 in order to immortalize these cells. After the selection with $\mathrm{G} 418$, a cell clone named $\mathrm{TEC}_{3}$ was obtained. RT-PCR, Western blot and immunocytochemical evaluation indicated that $\mathrm{TEC}_{3}$ cells differentiated from mouse ES cells still maintained the phenotype of vascular endothelial cells(Fig 1), and could proliferate continuously and be passaged for long-term in vitro[14].

Generally, $\mathrm{TEC}_{3}$ cells were cultured in DMEM medium containing $15 \%$ FCS. At the interval of 3 passages, $\mathrm{TEC}_{3}$ cells were recaptured in DMEM containing $100 \mathrm{ng} / \mathrm{ml} \mathrm{LIF,} 50 \mathrm{ng} / \mathrm{ml} \mathrm{VEGF}$ and $10 \mathrm{ng} / \mathrm{ml}$ bFGF to maintain endothelial cell phenotype.

Flow cytometry assay for DNA euploidy of $\mathrm{TEC}_{3}$ cells

$\mathrm{TEC}_{3}$ cells (the $17^{\text {th }}$ passage used as experimential material) and ES7 cells (the $9^{\text {th }}$ passage used as control) were collected respectively, fixed with $70 \%$ ethanol and stained routinely, then analyzed by FACS Caliber Flow cytometry for cell DNA euploid.

\section{Flow cytometry assay for $v W F$ expression in $\mathrm{TEC}_{3}$ cell}

$\mathrm{TEC}_{3}$ cells and ES7 cells were also used for the analysis of vWF expression. $\mathrm{TEC}_{3}$ cells and ES7 cells were collected respectively and stained with a rabbit anti-vWF antibody (1:50, Dako) for immunofluorescence by fluorescence activated cell sorter (FACS). The secondary antibody was goat anit-rabbit IgG-FITC (1:50). After the reaction was finished, $\mathrm{TEC}_{3}$ cells and ES7 cells were analyzed by FACS Caliber for the percentage of positive cells. Mouse bone marrow-derived endothelial cells (BMECs)[14] were used as positive control cells.

Isolation and culture of smooth muscle cells (SMCs)

Carotid arteries were harvested from New Zealand white rabbits, 
washed with phosphate buffered saline (PBS) for several times, then minced by scissors after adventitia was deprived. The tissue fragments were plated on the culture dishes (Falcon, $10 \mathrm{~cm}$ in diameter), with a distance around 1 2 mm among tissues pieces. Small amount of DMEM medium supplemented with $10 \%$ fetal bovine serum (FBS, Hyclone) and 100 units/ml penicillin and streptomycin (Gibco) was added, and the dishes were put in an incubator. After $4 \mathrm{~h}$ incubation at $37^{\circ} \mathrm{C}$ and $5 \% \mathrm{CO}_{2}$, the tissue fragments adhered to the dish well and then $10 \mathrm{ml}$ per culture dish of DMEM/FBS was added. After culture for another 6-7 days, some fibroblast-like cells grew out from the artery tissue pieces, and then culture medium was changed every 2-3 days.

Three weeks later, these fibroblast-like cells were grown near to confluence. Cells were then trypsined and passaged. Meanwhile, these cells were proved to be smooth muscle cells by immunocytochemical staining with an anti-smooth muscle actin antibody (Dako).

\section{Construction of tissue engineered tubular structure}

Polyglycolic acid (PGA) nonwoven fibers were purchased from Albany International Research Company, Inc (Albany, NY, USA). The PGA fibers were arranged into a sheet of $3 \times 0.5 \mathrm{~cm}$ in size(Fig 2) and sterilized by immersing in $75 \%$ alcohol for half an $\mathrm{h}$.

SMCs proliferated continuously in vitro. These cells were trypsinized when the number of cells reached $8 \times 10^{6}$, and then seeded onto a sterilized PGA sheet and left in an incubator at $37^{\circ} \mathrm{C}$, DMEM medium containing $10 \%$ FBS was added 4 hours later, culture medium was changed every day. After 1 week in vitro culture, the cellPGA construct was wrapped around a silicon tube ( $3 \mathrm{~mm}$ in diameter) (Fig 3), and then implanted subcutaneously into nude mice (Fig 4).

\section{Endothelialization of tubular structure}

After the tubular structure was implanted into nude mice for $6 \sim 8$ weeks, the constructed tube was exposed by a small incision (Fig 5), the silicon tube was pulled out and replaced by another silicon tube with smaller diameter( $2 \mathrm{~mm}$ in diameter), and then $2 \times 10^{6} \mathrm{TEC}_{3}$ cells suspended in $500 \mathrm{ml}$ culture medium were injected into the interspace between the silicon tube and engineered vessel, and the incision was sutured. Only culture medium $(500 \mu 1)$ was injected in control group. Engineered vessels were harvested in 5 days after $\mathrm{TEC}_{3}$ cells were injected. The harvested tubes were analyzed for gross observation (Figs 6,7,8). The harvested tissues were also embedded in paraffin, sectioned at $5 \mathrm{~mm}$ and then proceeded for histology and immunohistochemical analysis such as vWF and smooth muscle actin (SMactin) staining. Rabbit carotid artery was used as positive control for the staining of vWF.
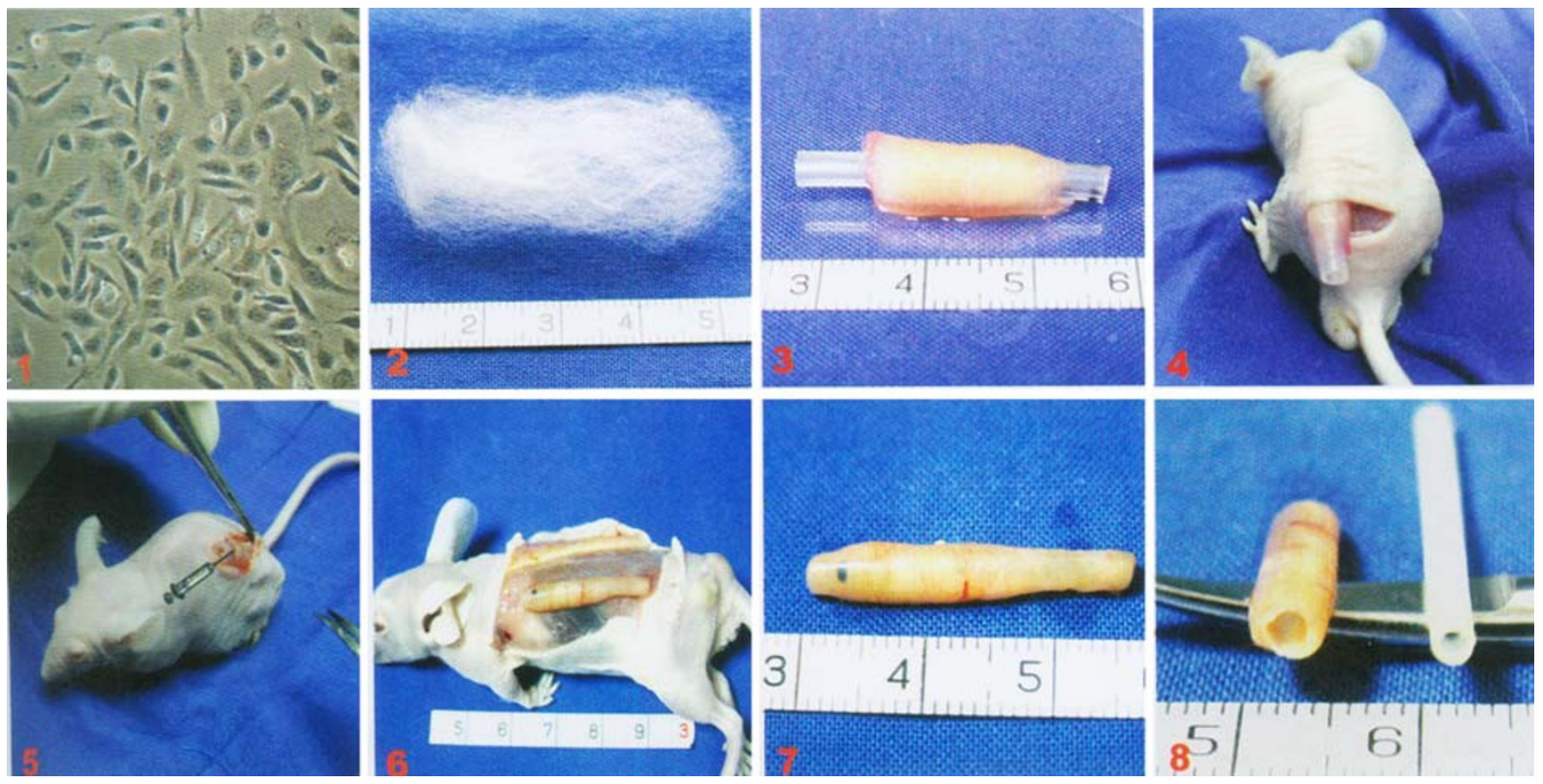

Fig 1. $\mathrm{TEC}_{3}$ cells (17th generations) Phase contrast. $200 \times$

Fig 2. The PGA fibers were fabricated into a sheet of $1.5 \times 3 \mathrm{~cm}$ in size.

Fig 3. SMCs-PGA was wrapped around a silicon tube (3 $\mathrm{mm}$ in diameter).

Fig 4. Tube wrapped with SMCs-PGA was implanted subcutaneously in nude mouse.

Fig 5. After the tubule implanted subcutaneous in nude mouse for 6 weeks, the tubular structure was exposed by a small incision and $\mathrm{TEC}_{3}$ cells were injected into the interspaces between the second silicone tube ( $2 \mathrm{~mm}$ in diameter $)$ and engineered vessel. (Arrow shown)

Fig 6. 5 days later, the engineered vessel was exposed in situ.

Fig 7. Tissue-engineered blood vessels with endothelial cells differentiated from ES cells showed yellowish pink in color.

Fig 8. Pulled out the tubule. Tissue-engineered blood vessel with lumen. 


\section{RESULTS}

\section{Characterization of TEC3 cells}

In vitro, $\mathrm{TEC}_{3}$ cells grew very well and maintained normal morphology of endothelial cells (data not shown) when examined at the $17^{\text {th }}$ passages. When compared with normal mouse ES7 cells by flow cytometry assay, $\mathrm{TEC}_{3}$ cells showed mainly euploidy in nature (Fig 9).

For endothelial cell marker vWF, FACS analysis showed that its expression (percentage of positive cells) in TEC $_{3}$ cells, ES7 cells (as negative control), and BMEC cells (as positive control) were $85.27 \%, 5 \%$, and $31 \%$ respectively (Fig 10). These results indicated that $\mathrm{TEC}_{3}$ cells (at the $17^{\text {th }}$ passage) still maintained the phenotype of endothelial cells.
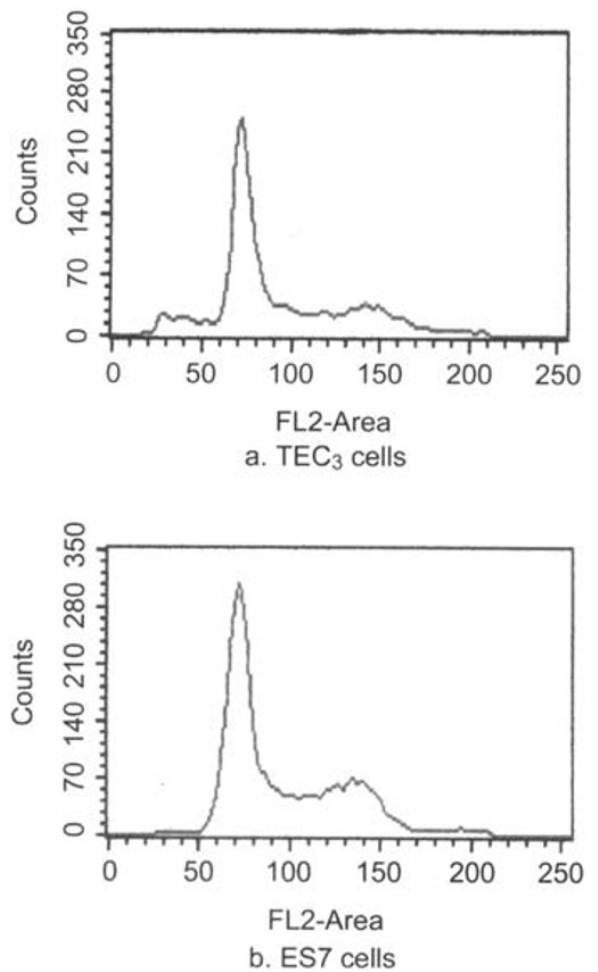

Fig 9. Flow cytometry assay for DNA euploidy of $\mathrm{TEC}_{3}$ cells (a) and ES7 cells (b).

\section{Blood vessel engineering}

After 1 week culture of several cubes of rabbit carotid arteries, fibroblast-like cells grew out around artery cubes (data not shown), and could reach confluence by 3 weeks (Fig 11). These cells were positive for SM a-actin by immunocytochemical staining (Fig 12a), but control group (dermal fibroblast cell) was negative (Fig 12b). Therefore, the cells derived from the artery cubes were smooth muscle cells (SMCs).

After SMCs were seeded onto PGA and cultured for
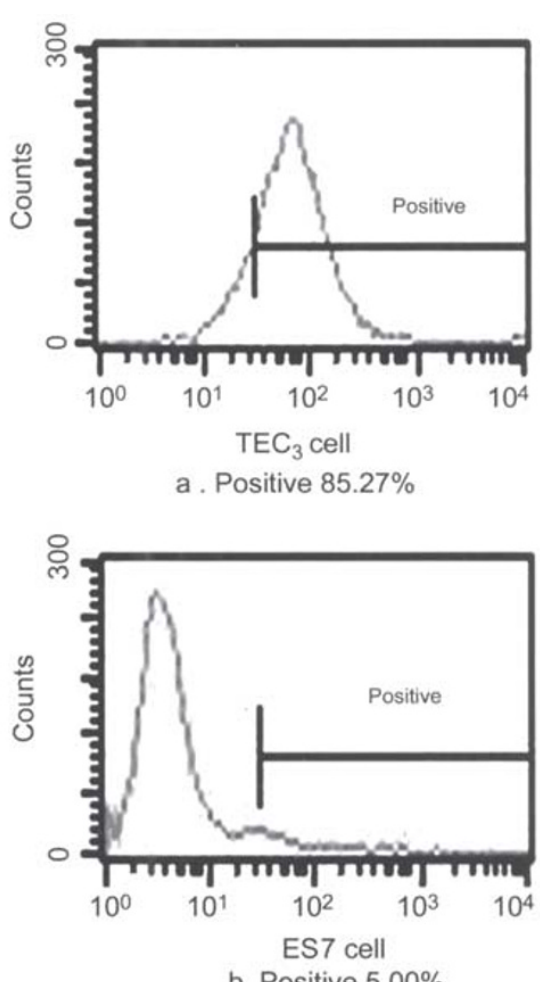

b. Positive $5.00 \%$

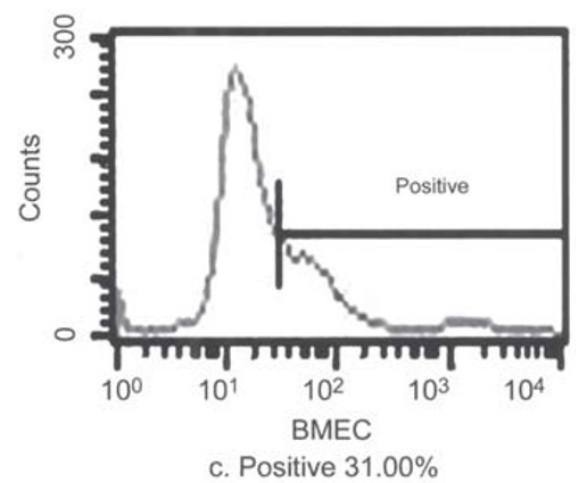

Fig 10. Flow cytometry assay for anti-vWF by fluorescent staining in $\mathrm{TEC}_{3}$ cells (a) ES7 cells (b) and BMEC (c). Percent positive cells are shown.

1 week, the cells grew very well, and secreted a lot of matrix proteins (Fig 13) and the cell-PGA construct formed a complex interwoven structure(Fig 14). Scanning electron microscope demonstrated that cells adhered to PGA fibers by 3 days (Fig 15), and the cells together with their matrix proteins became intermingled with PGA fibers by 7 days to form a continuous complex structure (Fig 16) .

The SMC tubes were harvested from nude mice after 5 day injection of the TEC 3 cells. The tissue-engineered blood vessels were yellowish pink in color (Fig 7) with a flat lumen according to the gross observation (Fig 8). A 

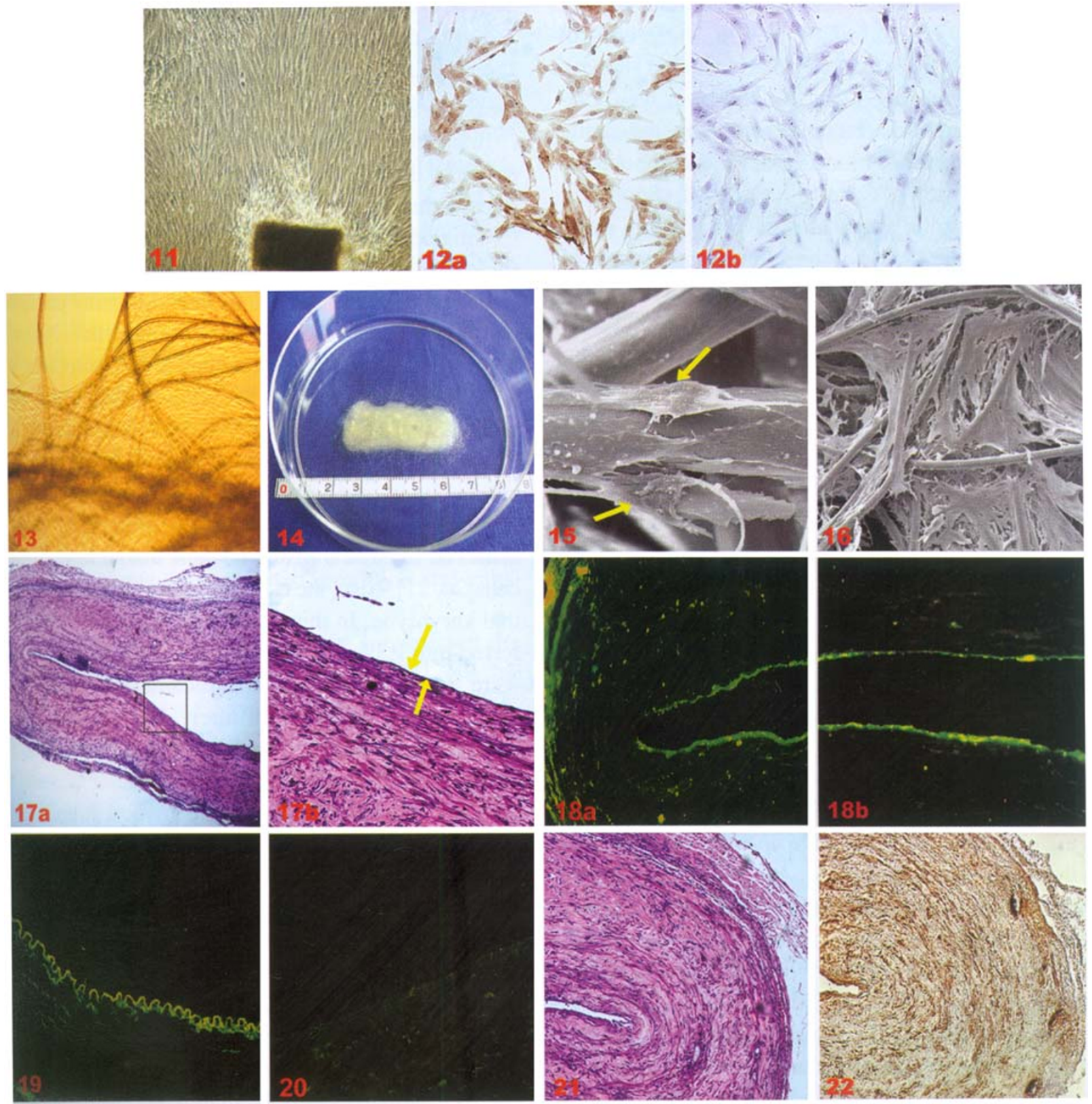

Fig 11. Cells from artery fragments cultured in vitro can be confluent by 3 weeks. Phase contrast $100 \times$

Fig 12a. SMCs were positive for smooth muscle actin in immunocytochemical neaction. $200 \times$

Fig 12b. Dermal Fibroblast cells were negative for smooth muscle actin in immunocytochemistry. $200 \times$

Fig 13. After SMCs were seeded onto PGA and cultured for 1 week, cells could grow very well, and secreted a lot of matrix proteins. Phase contrast $100 \times$

Fig 14. SMCs-PGA formed a complex interwoven structure.

Fig 15. Scanning electron microscope observation, cells were adhered to PGA fibers very well to form a sheet like structure by 3 days $780 \times$

Fig 16. SMCs and matrices became intermingled with PGA by 7 days so that spaces as seen in Fig 15 were no longer present. Scanning electron microscope $500 \times$

Fig 17. a, H.E staining of tubular structure. $100 \times$; b, Magnified part of H.E staining of tubular structure, a single layer of endothelial cells on the lumen surface. $200 \times$

Fig 18. a.b. A section of tubular structure showing immunofluorescent positive staining of endothelium to anti-vWF. $100 \times$

Fig 19. Endothelium layer of rabbit carotid artery showed immunofluorescent positive staining for anti-vWF. $100 \times$

Fig 20. The control group not injected with TEC3 cells gave negative reaction to vWF antibodies. $100 \times$

Fig 21. Wall of constructed blood vascular were composed with SMCs and arrayed in bundle. $100 \times$

Fig 22. Wall of constructed blood vascular showing positive immunohistochemical staining for SM-actin of smooth-muscle cells. $100 \times$ 
single cell layer lining at the luminal surface was observed by HE staining (Fig 17a,b). In control group no such cell layer was observed (data not shown). The tissue section showed that this cell layer was positive for vWF by immunofluorescent staining, indicating that the intimate layer of blood vessel was composed of $\mathrm{TEC}_{3}$ cells (Fig 18a,b). As a positive control, endothelial layer of rabbit carotid artery also gave a positive staining (Fig 19) similar to that of the experimental group. In negative control tube, no positive staining for vWF was found (Fig 20). SMCs of tissue engineered blood vessel arrayed in bundle. (Fig 21), and showed positive staining for SM a-actin (Fig 22). All these results indicated that PGA fibers were degraded after the SMC-PGA construct was implanted subcutaneously into nude mice for 6-8 weeks. In addition, tubular structures were composed with SMCs, which secreted a lot of matrices. The engineered vessels also contained an endothelium layer constructed by $\mathrm{TEC}_{3}$ cells.

\section{DISCUSSIONS}

The concept of tissue engineering was first proposed in the mid-1980s to solve the problem of donor shortage in tissue or organ transplantation[15]. It is a new area that involves cell biology, material and engineering sciences. The basic approach is to seed different kinds of living cells onto biodegradable polymer that can supply a three-dimensional scaffold. And when the polymer degrades in vivo, the proliferated seeded cells and their secreted extracellular matrix,together with other necessary infiltrated cells, eventually form a regenerated tissue that can replace the defected tissue. The seed cells play a very important role in tissue engineering. However, due to the aging of seed cells during in vitro expansion and the morbidity of donor site when harvesting tissue, a reliable source of seed cells remains a major concern in tissue engineering. The similar concern also exists in blood vessel engineering. Firstly, animals may face a lifethreaten when large vessels are harvested to isolate endothelial cells; secondly, the endothelial cells should be isolated by means of enzyme digestion, which may affect cell viability; and thirdly, the cells became aged when they are expanded in vitro.

Since mouse and human pluripotent embryonic stem (ES) cell lines have been successfully established from blastocysts in 1981 and 1998 respectively[16,17,7]. It has been reported that ES cells can differentiate into many kinds of committed cells by the induction with growth factors, cytokines or conditioned culture medium, and by gene manipulation[8,18,19], etc. Therefore, ES cells are expected to become a new source of seed cells for tissue engineering.

In our previous studies[8,14], ES cells have been induced to differentiate into endothelial cells by RA and transforming growth factor $\beta_{1}\left(\mathrm{TGF}-\beta_{1}\right)$, but these differentiated endothelial cells were not able to proliferate for a long time in vitro. Normal human cells undergo a finite number of cell divisions and ultimately enter into a non-dividing state called replicate senescence. It has been proposed that telomere shortening is the molecular clock that triggers senescence. Previous studies showed that when retinal pigment epithelial cells and foreskin fibroblasts were transfected respectively with the vector encoding the human telomerase catalytic subunit (hTCS), more cells doubling (at least 20 doubling) could be achieved in these transfected cells than non-transfected cells[20,21].Also, the established two cell lines had normal karyotype. In this study, hTERT cDNA was transferred into ES7 cells to give $\mathrm{TEC}_{3}$ cells and these cells were able to maintain the characteristics of endothelial cells and to proliferate infinitely[14]. Flow cytometry analysis demonstrated that there were $85.27 \% \mathrm{TEC}_{3}$ cells (Fig 10) that still expressed vWF after expansion to passage 17 , so these cells could provide a source as seed cells for constructing the endothelium of tissue engineered blood vessel. Also, the results from flow cytometry assay for DNA euploidy (Fig 9) and karyotype analysis (data not shown) indicated that the $\mathrm{TEC}_{3}$ cells belong essentially to normal cells as compared with parent ES7 cells.

After implantation of the tubular structure in nude mice for 6-8 weeks, we exposed the tubular structure with a small incision, replaced the silicon tube with another one in smaller diameter and injected $2 \times 10^{6} \mathrm{TEC}_{3}$ cells into the interspace between silicon tube and engineered vessel. Five days later, the luminal surface of harvested vessel was shown to be positive for vWF staining. As we know, the normal artery wall consists mainly of endothelial cells (internal layer), smooth muscle (medial layer), and other types cells (adventitia layer). Our goal is to engineer a blood vessel with a structure similar to that of normal blood vessel.

The early 'model of engineering blood vessel' consisted of a multi-layered structure including animal collagen and cultured bovine endothelial cells, smooth muscle cells and fibroblasts[2]. However, these blood 
vessel is too weak to withstand pressure. So, many methods have been tried to improve the mechanical strength of tissue engineered blood vessel[3,4].In 1998, Shinoka[4] seeded SMCs and ECs onto PGA to produce a 'living' pulmonary artery conduit. The results showed that these kinds of tissue engineered grafts were potent after 11 weeks. In our lab, the method is similar to Shinoka's; Liu et al. [6] found that none of polymer scaffold was remained after the tubular structure of cellPGA construct had been implanted into nude mice subcutaneously for 6 weeks. So, in the present work, we harvested the engineered vessel wall at 6-8 weeks and seeded $\mathrm{TEC}_{3}$ cells into the lumen for another 5 days. From a total of constructed 5 tissue engineered blood vessels, we found that one of them was consisted of multi-layer $\mathrm{TEC}_{3}$ cells, which even blocked a few parts of tubule lumen. This phenomenon was probably correlated with the unequal distribution of $\mathrm{TEC}_{3}$ cells, due to the slope of tube and gravity, in the interspace between the smaller silicon tube and engineered vessel.

In summary, we have successfully constructed the tissue engineered blood vessels with endothelial cells differentiated from mouse ES cells, and our results demonstrated for the first time the possibility, that ES cells can be used as the seed cells for blood vessel engineering. But there is a long way to go to use this kind of seed cells for clinical application and further more detailed research needs to be performed.

\section{ACKNOWLEDGEMENTS}

This work was supported by the national "973" tissue engineering project of China (G1999054300), Shanghai Science and Technology Development Foundation (03DJ14021). The authors are very thankful to Prof. Zhen YAO (Laboratory of Embryonic Stem Cell, Institute of Biochemistry and Cell Biology, Shanghai Institutes for Biological Sciences, Chinese Academy of Sciences) for his valuable discussions and revisal of this manuscript.

\section{REFERENCE}

1 Niklason L, Gao T, Abbott W.M, et.al. Functional arteries grown in vitro. Science 1999; 284:489-93.

2 Weinberg CB, Bell E. A blood vessel model constructed from collagen and cultured vascular cells. Science 1986; 231:337-40.

3 L'Heureux NL, Germain L, Labbe R, et al. In vitro construction of a human blood vessel from cultured vascular cells: A mor phologic study. J Vasc Surg 1993; 17:499-502.

4 Shinoka LE, Gao J, Abbott WM, et al. Creation of viable pulmonary artery autografts through tissue engineering. J Thorac Cardiovasc Surg 1998; 115:536-45.

5 L'Heureux N, Paquet S, Labbe R, et al. A completely biological tissue-engineered human blood vessel. FASEB J 1998; 12:47-55.

6 Liu DL, Liu Y, Liu W, et. al. Creating small-caliber artery through tissue engineering in nude mice and dogs, 65A, 47th Annual Meeting of plastic Surgery Research Council.2002, Boston, Massachusetts.

7 Thomson JA, J.Iaskovitg-Eldor, Shapiro SS, et al. Embryonic stem cell lines derived from human blastocysts. Science 1998; 282: 1145-7.

8 Tsung HC, Xia SH, Xu LX, et al. Expression of exogenous porcine transforming growth factor beta-1 gene in ES cells and its effect on their differentiation in vitro. Acta Biologiae Exp Sinica 1995; 28:173-189.

9 Hirashima M, Kataoka H, Nishikawa S and Matsuyoshi N. Maturation of embryonic stem cells into endothelial cells in an in vitro model of vasculogenesis. Blood 1999; 93:1253-63.

10 Balconi G, Spagnuoeo R and Dejana E. Development of endothelial cell lines from embryonic stem cells: A tool for studying genetically manipulated endothelial cell in vitro. Arterioscler Thromb.Vasc Biol 2000; 352:1443-51.

11 Levenberg S, Golub JS, Amit M, et al. Endothelial cells derived from human embryonic stem cells. PNAS 2002; 99:4391-406.

12 Paul ph A, Joosten H, Verhagen HJM, et al. Thrombogenesis of different cell types seeded on vascular grafts and studied under blood-flow conditions. J Vasc Surg 1998; 28:1094-103.

13 Luo XJ, Wu GY. The development on small-caliber prosthetic graft. Chin J clin Thorac cardiovasc Surg 2001; 8:193-6.

14 Shen G, Cong XQ (syn.:Tsung HC), Du ZW, et al. Immortalization of endothelial cells differentiated from mouse embryonic stem cells. Acta Biol Exp Sinica 2002; 35:218-28.

15 Langer R, Vacanti JP.Tissue engineering. Scince 1993; 260:920-6.

16 Evans MJ and Kaufman M. Establishment in culture of pluripotential stem cells from mouse embryos. Nature 1981; 292: 151-6.

17 Martin GR. Isolation of a pluripotent cell line from early mouse embryos cultured in medium conditioned by teratocareinoma stem cells. Proc Natl Acad Sci USA 1981; 78:7634-8.

18 Buttery LDK, Bourine S, Xynos DJ, et al. Differentiation of osteoblasts and in vitro Bone formation from murine embryonic stem cells. Tissuue Engineering 2001; 7:89-97.

19 Nakayama N, Duryee D, Manoukian R et al. Macroscopec cartilage formation with embryonic stem cell derived mesodermal progenitor cells. J Cell Science 2003; 116:2015-28.

20 Bodnar AG, Ouellette M, Frolkis M, et.al. Extension of life span by introduction of telomerase into normal human cells. Science 1998; 279:349-52.

21 Vagiri $\mathrm{H}$ and Benchimol S. Reconstitution of telomarase activity in normal human cells leads to elongation of telomerase and extended replicative life span. Current Biology 1998; 8:279-82. 